\title{
Dipolar Rings of Microscopic Ellipsoids: Magnetic Manipulation and Cell Entrapment
}

\author{
Fernando Martinez-Pedrero ${ }^{1,2}$, Andrejs Cebers $^{3}$, and Pietro Tierno ${ }^{1,2 *}$ \\ ${ }^{1}$ Departament de Física de la Matèria Condensada, Universitat de Barcelona, Barcelona, Spain. \\ ${ }^{2}$ Institut de Nanociéncia i Nanotecnologia, IN ${ }^{2}$ UB, Universitat de Barcelona, Barcelona, Spain. \\ ${ }^{3}$ Faculty of Physics and Mathematics, University of Latvia, Zellu 23, LV-1002, Riga.
}

(Dated: August 30, 2016)

\begin{abstract}
We study the formation and dynamics of dipolar rings composed by microscopic ferromagnetic ellipsoids, which self-assemble in water by switching the direction of the applied field. We show how to manipulate these fragile structures and control their shape via application of external static and oscillating magnetic fields. We introduce a theoretical framework which describes the ring deformation under an applied field, allowing to understand the underlying physical mechanism. Our microscopic rings are finally used to capture, entrap and later release a biological cell via magnetic command, i.e. performing a simple operation which can be implemented in other microfluidic devices which make use of ferromagnetic particles.
\end{abstract}

\section{INTRODUCTION}

A ring is a fascinating one-dimensional topological structure which encircles a finite two-dimensional region of the space. When deformed, a ring may reduce its enclosed surface area and acquire a complex shape resulting from competition between compression and bending rigidity. At the microscale, there are different ways to assemble colloidal particles into rings, such as by using electric fields [1, laser beams [2, 3, capillary forces [4, 5] or time-dependent magnetic fields [6 9]. In contrast, in the absence of external forces, rings formed by spontaneous self-assembly are more difficult to observe. When the particles interact via isotropic and attractive pair potentials, the formation of compact clusters is favored over open, ring-like structures 10. On the other hand, anisotropic pair interactions like those arising from induced moments promote chaining along a defined direction [11, 12. Dipolar rings have been observed with magnetized spheres [13, 14] when balancing any external field, and with Janus-magnetic microrods [15], when inverting the direction of the applied field. Minimization of the system energy under such conditions shows that the equilibrium configuration of dipolar particles corresponds to closed rings [13, 16, 17. While the formation of dipolar rings [18 28] and their rupture under an external field [15, 19] have received attention in the past, no work demonstrates the possibility to manipulate these fragile assembly without breaking them, and in such a way to perform functional tasks.

Here we study the formation and dynamics of dipolar rings self-assembled from microscopic hematite ellipsoids, ferromagnetic and anisotropic particles with a magnetic moment perpendicular to their long axis. We manipulate these self-assembled structures by using an external field and present a theoretical framework that explains the ring deformation and dynamics under the applied field. Finally, we use these rings to entrap and later release micro-objects such as a biological cell, in a fluidic

\footnotetext{
* ptierno@ub.edu
}

environment, extending the potentiality of our technique to applications in fluidic microsystems based on precise micro-encapsulation.

\section{EXPERIMENTAL METHOD AND RING FORMATION}

We synthesize monodisperse hematite ellipsoids following the method developed by Sugimoto et al. 29]. Inspection of scanning electron microscopy images of the particles reveals that they are prolate ellipsoids with major (minor) axis equal to $a=1.80 \mu \mathrm{m}(b=1.33 \mu \mathrm{m}$ resp.). After synthesis, the ellipsoids are dispersed in pure water (milliQ, Millipore), stabilized with sodium dodecyl sulfate and the $\mathrm{pH}$ is adjusted to $8.5-9.5$. The particles sediment close to a glass plate, where they remain quasi two-dimensional confined due to gravity. To visualize the particle dynamics, we use a upright light microscope (Eclipse Ni, Nikon), and record their position with a CCD camera (Scout scA640-74f, Basler) working at 50 fps. We apply different external magnetic fields by using a custom-made triaxial coil system connected either to a power supply (EL 302RT, TTi), when needed to generate a DC field, or to a wave generator (TTi-TGA1244, TTi) feeding a power amplifier (IMG STA- 800, Stage Line) when needed to generate AC magnetic fields [30].

In the absence of any applied field, the ellipsoids rapidly assemble into a ribbon-like structure due to the presence of a small permanent moment $\vec{m}$ in the particles, perpendicular to the ellipsoid long axis. Once formed, the ribbon does not orient randomly, but aligns in average along the direction given by the earth magnetic field, $H_{e} \sim 0.5$ Oe. As shown in Fig.1, by applying a small field of amplitude $H=2 H_{e}$ in the opposite direction, we are able to form a dipolar ring from a ribbon configuration, see Video 1. The ribbon slowly deforms assuming an hairpin-like shape, and its two free ends approach each other, and finally join forming a ring. The kinetic pathway described in Fig.1 is only one possible route towards ring formation. We also find that after applying the small external field $H$, the ribbon can break into two rings via 


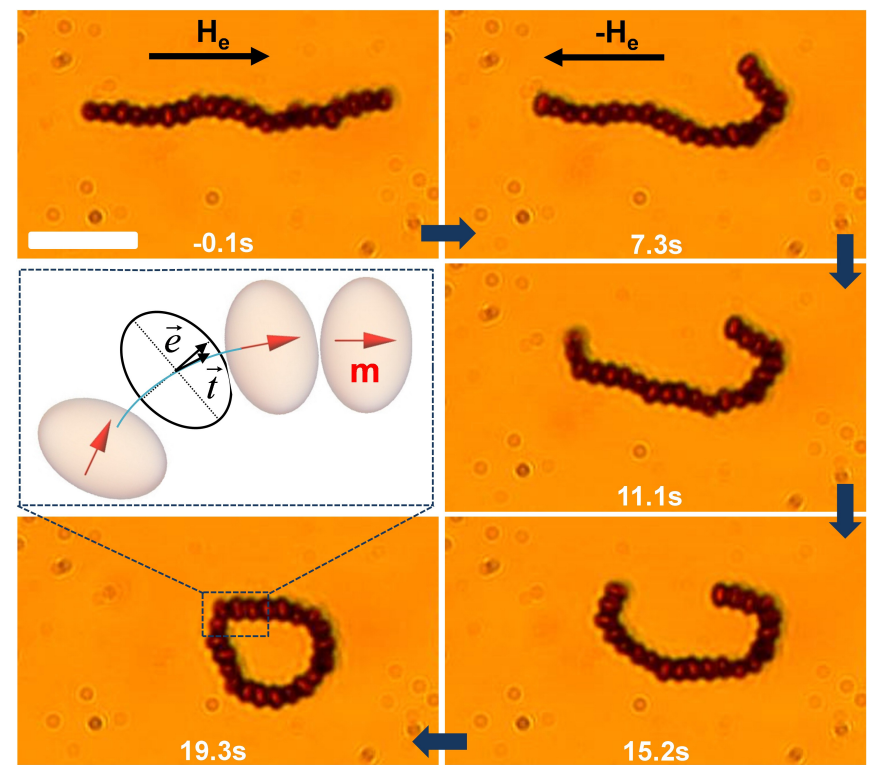

FIG. 1. Sequence of images showing the formation of a colloidal ring composed of $N=21$ hematite ellipsoids. The initial image shows a ribbon oriented along the direction of the earth magnetic field $H_{e}$. At $t=0$, a static field $H=-2 H_{e}$ is applied and the ribbon closes into a ring after 19.3s (see Video 1). Scale bar is $10 \mu \mathrm{m}$. The schematic in the middle left illustrates four ellipsoids with magnetic moments $\vec{m}$ perpendicular to their long axis and the vectors $\vec{t}$ and $\vec{e}$.

an S-shape deformation, when the two ends of the chain curve in opposite directions, as observed with ferromagnetic Janus rods [15.

\section{ANALYSIS OF RING DEFORMATION}

We control the shape of the ring by further imposing an additional static field in the particle plane. As shown in Fig.2(a), the ring shrinks along the applied field. In contrast, the ribbons orient along the applied field, as shown in Video 2. The applied field forces the magnetic moments of the particles to deviate from the tangent direction of the ring, described by the vector $\vec{t}$. Thus, the magnetic energy will depend upon the angle between $\vec{t}$ and the applied field, $\vec{H}=H \vec{h}$. Given the small anisotropy of our hematite particles, with an aspect ratio of $\sim 0.74$, we describe the ring as an ensemble of interacting spheres characterized by a point dipole at the center. We write the total magnetic energy per unit length of the ring as

$$
e_{m}=-M \vec{e} \cdot \vec{H}-\frac{1}{2} \Delta N M^{2}(\vec{e} \cdot \vec{t})^{2},
$$

being $M$ the magnetization per unit length, $\vec{e}$ the unit vector along the local particle magnetization which may vary along the contour of the ring, $\Delta N=6 K_{N}^{\prime} / d^{2}$ the demagnetization factor, $d$ the particle diameter and
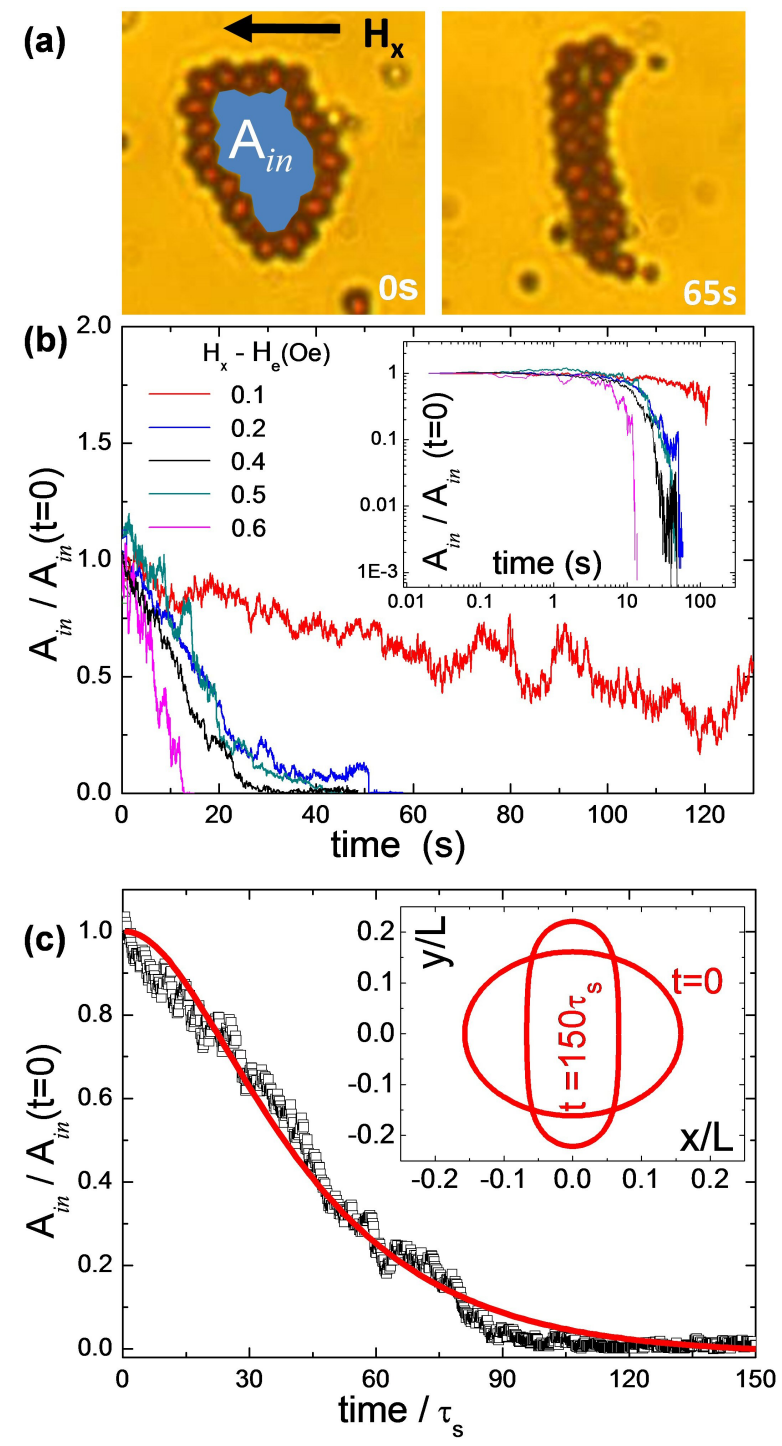

FIG. 2. (a) Deformation of a colloidal ring composed by $N=20$ ellipsoids when subjected to a static field $H_{x}$. See also Video 2 that shows a smaller ring in the proximity of a ribbon. (b) Normalized area enclosed by the ring versus time for different applied magnetic fields. $A_{\text {in }}$ denotes the internal area of the ring. Inset shows the same plot but in a $\log$-log scale. (c) Normalized area versus re-scaled time $t / \tau_{s}$ $\left(\tau_{s}=3.5 \mathrm{~s}\right)$ for a dipolar ring of $N=20$ particles compressed via application of a constant field $H_{x}-H_{e}=0.4$ Oe. Scattered points are experimental data, while continuous red line is the result of the numeric integration of Eq.6. Inset shows the calculated ring contour at different rescaled times.

$K_{N}^{\prime}=\frac{1}{4} \sin ^{3}(\pi / N) \sum_{i=2}^{N} \frac{1-\cos (2 \pi(i-1) / N)}{\sin ^{5}(\pi(i-1) / N)}$, see the Appendix A for a detailed derivation. We note here that the particle diameter $d$ used in the model is replaced in our calculations by the minor axis of the ellipsoid $b$. As demonstrated in the Appendix, Eq.1 is rather general, and can be equally applied to both a chain or a ring of dipoles. In the limit of small applied fields, Eq.1 can be 
transformed (see Appendix B) to:

$$
e_{m}=-M H \vec{t} \cdot \vec{h}+\frac{H^{2}}{2 \Delta N}(\vec{t} \cdot \vec{h})^{2} .
$$

Since $\vec{t}=d \vec{r} / d l$, the first term in Eq.2 vanishes after integration along the closed centerline of the ring and only the second term gives a non-vanishing contribution to the energy of the ring. Further, in the limit of small fields, the ring has a negative anisotropic magnetic susceptibility $\chi_{\|}-\chi_{\perp}=-1 / \Delta N\left(\chi_{\|}, \chi_{\perp}\right.$ denote parallel and perpendicular to the local tangent direction respectively). Therefore there is a tendency to increase the length of the elements of the ring which are perpendicular to the external field. Considering the ring in the frame of the Kirchoff model of elastic rods, with added magnetic energy terms 32, the torque balance equation per unit length of the ring reads

$$
\frac{d \vec{T}}{d l}+[\vec{t} \times \vec{F}]+M H[\vec{t} \times \vec{h}]-\frac{H^{2}}{\Delta N}(\vec{t} \cdot \vec{h})[\vec{t} \times \vec{h}]=0,
$$

where $\vec{T}$ and $\vec{F}$ are the torque and the force in the crosssection of the ring respectively. The total force is given by, $\vec{F}=\vec{F}_{e}+\vec{F}_{m}$. Here $\vec{F}_{e}$ includes the contribution of the elasticity,

$$
\vec{F}_{e}=-A \frac{d^{3} \vec{r}}{d l^{3}}+\Lambda \frac{d \vec{r}}{d l},
$$

where $A$ is the bending modulus, the Lagrange multiplier $\Lambda$ accounts for inextensibility of the rod [33], and the magnetic shearing force is given by

$$
\vec{F}_{m}=-M H \vec{h}+\frac{H^{2}}{\Delta N}(\vec{t} \cdot \vec{h}) \vec{h} .
$$

Since in Eq.5 the first term is homogeneous, it does not enter in the equations of motion. The equation of motion for the ring reads:

$$
\zeta_{e} \vec{v}=\frac{d \vec{F}_{e}}{d l}+\frac{H^{2}}{\Delta N} \vec{h}\left(\frac{d^{2} \vec{r}}{d l^{2}} \cdot \vec{h}\right),
$$

where $\vec{v}$ denotes the velocity of the element of the ring, $\zeta_{e}=4 \pi \eta$ is the isotropic hydrodynamic drag coefficient of the ring per unit contour length and $\eta$ the viscosity of water. The total energy functional is given by:

$$
E=\frac{A}{2} \int\left(\frac{d \vartheta}{d l}\right)^{2} d l+\frac{H^{2}}{2 \Delta N} \int \cos ^{2} \vartheta d l,
$$

being $l$ the natural parameter along the ring contour, $\vec{t}=(\cos \vartheta, \sin \vartheta)$ and $d \vartheta / d l=-1 / R$ the ring curvature. Minimizing Eq.7 gives the Euler-Lagrange equation

$$
A \frac{d^{2} \vartheta}{d l^{2}}+\frac{H^{2}}{\Delta N} \cos \vartheta \sin \vartheta=0,
$$

written assuming an inextensible ring. Starting from this equation, it can be shown (Appendix C) that the shape of the ring is described by the set of equations (here, the contour length $l$ is scaled with the total length of the ring $L)$ :

$$
\frac{d x}{d l}=c n\left(\sqrt{\frac{C_{m}}{k^{2}}} l, k\right) ; \quad \frac{d y}{d l}=s n\left(\sqrt{\frac{C_{m}}{k^{2}}} l, k\right),
$$

written in a dimensionless form. Here $c n(u ; k)$ and $s n(u ; k)$ are the Jacobi elliptic functions of the first and second kind, $u$ is the argument and $k$ the modulus, $k^{2}=H^{2} /\left(2 \Delta N C_{1}\right), C_{1}$ is the first integral of Eq.8 and $C_{m}$ is the magnetoelastic number defined as

$$
C_{m}=\left(\frac{L}{d}\right)^{2} \frac{H^{2}}{\left(m / d^{3}\right)^{2}} \frac{1}{3 K_{N}^{\prime}},
$$

where the bending modulus of the ring is only given by the magnetic dipolar interactions according to $A=$ $m^{2} /\left(2 b^{2}\right)$ 34. The shape of the ring can be calculated by numerical integration of Eq.6.

\section{EXPERIMENTAL RESULTS}

In order to validate our theoretical model, we have performed a series of experiments by measuring the area enclosed by a ring $A_{i n}$ as it reduces due to the applied field $H_{x}$, at different field amplitudes. These realizations are shown in Fig.2(b), which reports the normalized internal area of the rings $A_{i n} / A_{i n}(t=0)$ as it reduces with time. A minimum field of $H_{x}-H_{e}=0.10$ is required to compress the ring. For field strengths higher than $H_{x}-H_{e}=0.2 \mathrm{Oe}$, we find that ring area reduces within $60 \mathrm{~s}$. In the range of values between 0.2 and $0.5 \mathrm{Oe}$, the dynamics of the ring deformation is difficult to distinguish, as shown in the inset in Fig.2(b). Thermal fluctuations make difficult to resolve the small differences in the shrinking dynamics of the rings, in the narrow range of applied fields. At fields larger than $H_{x}-H e=0.6 \mathrm{Oe}$, the ring structures break during the shrinking process. In Fig.2(c) we compare the experimental data for one field $H_{x}-H_{e}=0.4 \mathrm{Oe}$ with the theoretical predictions, and rescaled the time by $\tau_{s}=\tau_{e} \cdot 10^{-6} \mathrm{~s}$, where $\tau_{e}$ is the characteristic elastic deformation time used as adjustable parameter. The continuous red line fitting the experimental data in Fig.2(c) is obtained by numerically solving Eq.6, following the numerical approach described in 33. The equilibrium shapes calculated according to Eqs.9 coincide with the shape obtained from the numerical integration of Eq.6. We use a value of $C_{m}=557.7$ assuming $K_{N}^{\prime} \sim 1, d=b$ and a permanent moment of the ellipsoid given by $m=2.3 \cdot 10^{-13} \mathrm{emu}$, as previously measured from the reorientation dynamics of an individual ellipsoid [35. The good agreement with the experimental data reported in Fig.2(c) is obtained using a value of $\tau_{e}=3.5 \cdot 10^{6} \mathrm{~s}$. On the other hand, we can independently estimate this parameter, by assuming that the bending modulus is given only by dipolar interactions, 

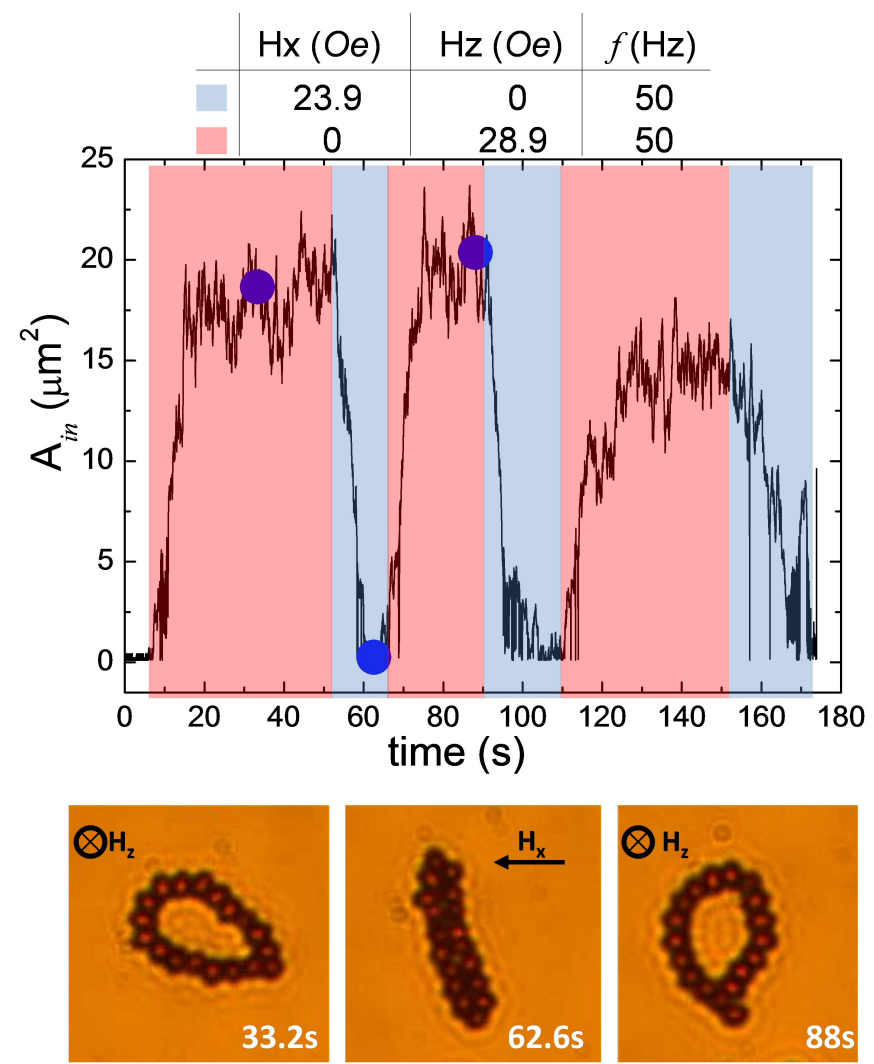

FIG. 3. Time evolution of the area $A_{\text {in }}$ of a dipolar ring composed of $N=18$ particles and subjected to a $50 \mathrm{~Hz}$ oscillating magnetic field applied either perpendicular to the ring $\left(H_{z}\right)$ or in the plane of the ring $\left(H_{x}\right)$. The filled scattered points indicate the location of the snapshots displayed at the bottom, which show the ring deformations.

$A=m^{2} / 2 b^{2}=1.5 \cdot 10^{-18} \mathrm{erg} \cdot \mathrm{cm}$. We thus find a characteristic timescale given by $\tau_{e}=\zeta_{e} L^{4} / A=4.2 \cdot 10^{6} \mathrm{~s}$, which is very close to the experimental value. Moreover, the persistence length of the chain can be estimated as the ratio $l_{p}=A / k_{B} T \cong 0.3 \mu \mathrm{m}$, being $k_{B}$ the Boltzmann constant and $T=293 \mathrm{~K}$ the experimental temperature. The small value of the persistence length obtained, smaller than the particle's size, may result from neglecting other attractive forces that increase the ring compactness. A small persistence length is consistent with the experimental observation that these rings are rather fragile, and easily deform due to thermal fluctuations. Effectively, we find that the these rings can easily break when the applied constant field exceeds the value of $\sim 0.6$ Oe.

The self-assembled microscopic rings studied here are kept only by dipolar forces, thus they can be easily broken by small static fields. However, we find that an oscillating field applied perpendicular to the plane of the ring, is able to stabilize the ring shape even having a much large amplitude. An oscillating field can be used to open a ring that has been previously closed, thus making completely reversible the induced deformation. These features are shown in Fig.3, which displays the controlled compression and expansion of one colloidal ring subjected to an oscillating magnetic field applied perpendicular to the ring, $H_{z}$ (expansion), or in the ring plane, $H_{x}$ (compression). We note here that the expansion of a dipolar ring cannot be achieved by using a simple static field since the latter will force the magnetic moments of the ellipsoids to orient along the field. In this situation, all moments in the rings will be perpendicular to the center-to-center distance, giving rise to a net repulsive dipolar interaction between them. In contrast, for an applied field oscillating faster than the individual reorientation of the ellipsoids, the particle moment is unable to follow the direction of the field. As a consequence, the moments orient perpendicular to the direction of the applied field [35 37]. Thus, for an oscillating field perpendicular to the ring plane, the moments will point along the plane of the ring, a situation which is favourable to the ring formation, similar to the configuration shown in Fig.1.

\section{CARGO ENTRAPMENT AND TRANSPORT.}

Beside the interest in studying self-assembly processes of magnetic particles [15, dipolar rings can also be used for lab-on-a-chip operations, such as to entrap and later release microscopic objects via external command. We demonstrate both features in Fig.4 (Video 3- Video 5), where a ribbon forms a ring which entraps one yeast cell within its boundaries. Before the trapping, and in order to approach the cell, the ribbon was translated at a constant speed of $\langle v\rangle=0.6 \mu \mathrm{ms}^{-1}$ upon application of an external precessing magnetic field 38. given by, $\vec{H} \equiv\left(H_{x}, H_{y} \cos (2 \pi f t), H_{z} \sin (2 \pi f t)\right)$, with frequency $f=50 \mathrm{~Hz}$, and amplitudes $H_{x}-H_{e}=0.3 \mathrm{Oe}$, $H_{y}=27.4 \mathrm{Oe}$ and $H_{z}=3.5 \mathrm{Oe}$. The applied field performs a conical precession around the $x$-axis; the static component $H_{x}$ and the oscillating component $H_{y}$ orient the ribbon along the $x$-direction and keep the particles attached to the ribbon. The rotating component induces the rotational motion of the individual ellipsoids. The ribbon thus translates in the perpendicular $(y-)$ direction, due to the hydrodynamic coupling of the rotating particles with the close glass surface [39]. This mechanism is similar to the propulsion of ensemble of paramagnetic colloids recently reported by Casic et al. [0]. Using this strategy, the ribbon is transported close to the cell from any location of the plane, see Video 3. After that, the precessing field is switched off, and a static field $H_{x}=0.7 \mathrm{Oe}$, applied contrary to $H_{e}$, is used to form a ring which complete encircles the cell after 16.2s, Fig.4(a) (Video 4). Once captured, the cell performs small thermal fluctuations within the magnetic ring, but it remains stably confined over time. In order to release the cell, without dismantling the magnetic structure, we compress the ring by applying a static magnetic field $H_{2}=0.2 \mathrm{Oe}$ along the $y$-direction, Fig.4(b) 
(a)

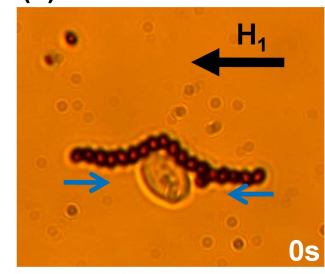

Cell entrapment
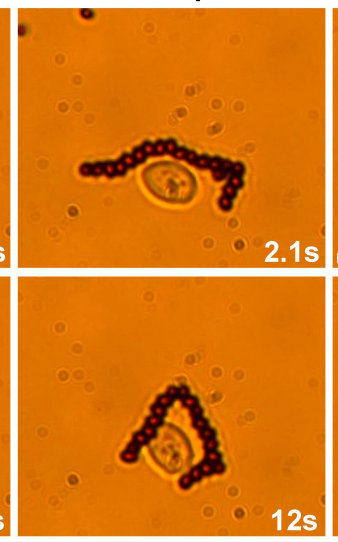

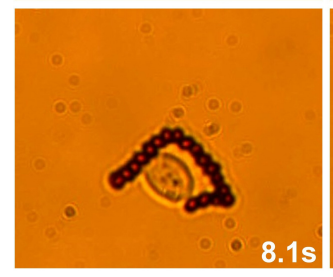

(b)

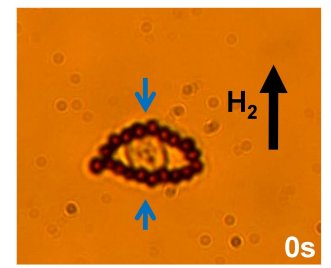

Cell release

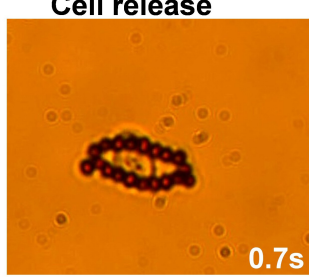

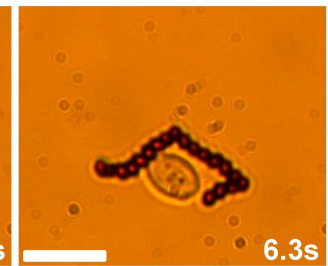
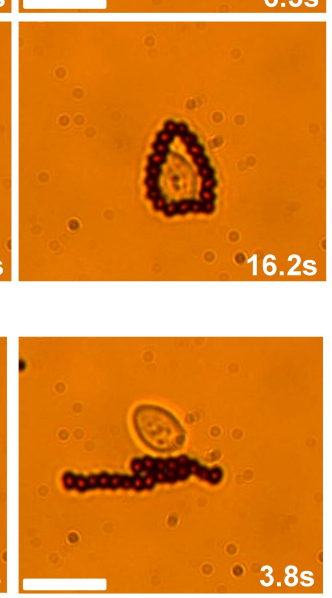

(c) Cargo transport

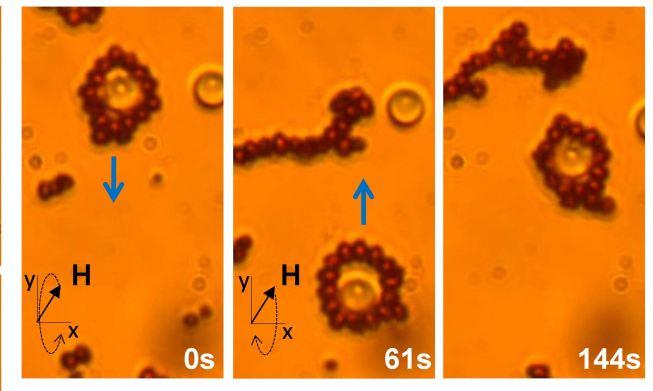

(d)

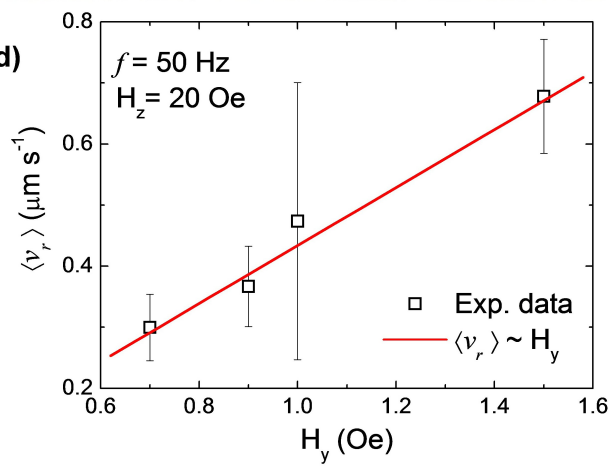

FIG. 4. (a) Sequence of images showing the entrapment of a yeast cell by a ribbon composed of 19 ellipsoids. Under a static field with amplitude $H_{1}=-2 H_{e}$ the ribbon encircles the cell after $16.2 s$ (Video 4). (b) Images showing the release of the cell upon application of a static field $H_{2}=0.2 \mathrm{Oe}$ which compresses the colloidal ring (Video 5). Scale bars are $10 \mu \mathrm{m}$ in both sequences. (c) Transport of a $3 \mu \mathrm{m} \mathrm{SiO} 2$ particle entrapped in a ring via application of a precessing magnetic field $\vec{H} \equiv\left(H_{x}, H_{y} \cos (2 \pi f t), H_{z} \sin (2 \pi f t)\right)$ with frequency $f=20 \mathrm{~Hz}$ and amplitudes $H_{x}-H_{e}=0.1 \mathrm{Oe}, H_{y}=0.7 \mathrm{Oe}$ and $H_{z}=7.10$. After $t=61 \mathrm{~s}$ the field polarity is inverted, and the ring changes its direction of motion (Video 6). (d) Average speed of the ring $\left\langle v_{r}\right\rangle$ versus amplitude of the in-plane field $H_{y}$ for a ring transporting the silica cargo. The continuous line denotes a liner fit to the experimental data.

(Video 5) The ring reduces its size and after $0.7 s$ the cell is squeezed off from the top part to be finally completely expelled from the ring. This operation is reversible, since the ribbon of particles can be transported again close to another microscopic object, where it can be transformed back into a ring to encircle it. When all the microscopic entities are two-dimensionally confined by gravity on the glass surface, the presence of the ring prevents future encounters between the cell and its neighbors. However, the cell is not protected in the third dimension. Our dipolar rings can be used to create a "protective" environment around a biological object when the system is two-dimensionally confined.

While the capture and the release have been all made by using uniform external fields, we have found that the rings can be also slowly transported by applying rotating fields, a further functionality which could be employed to translate micro-objects entrapped within this structure. In particular, in Fig.4(c) we show the transport of a $3 \mu \mathrm{m} \mathrm{SiO}_{2}$ particle which was previously entrapped in a ring composed of $N=15$ ellipsoids. The ring propulsion was induced by applying an external rotating magnetic field in the $(y, z)$ plane, perpendicular to the static field $H_{x}$. As shown in the corresponding Video 6 , the rotating field forces the ellipsoids to perform a rotational motion around their short axis. Similar to the translation of the linear ribbon, the mechanism of motion of the ring results from the rotational movement of the composing ellipsoids close to the glass substrate. During the motion of the ring, the trapped particle is unable to escape from the ring boundaries, and it is transported along the glass plate at a constant average speed $\left\langle v_{r}\right\rangle=0.59 \mu \mathrm{m}^{-1} \mathrm{~s}$. The transport process occurs without the dis-assembly of the ring structure during its motion, and reverting the polarity of the rotating field allows us to change direction of motion. Moreover, we find that the ring speed can be well controlled by tuning the amplitude of the in-plane field $H_{y}$, Fig.4(d). In the range of the field strengths here employed, we find that $\left\langle v_{r}\right\rangle$ scales linearly with $H_{y}$, reaching a speed up to $0.7 \mu m s^{-1}$ for $H_{y}=1.5 \mathrm{Oe}$. Compared to the propulsion of the linear ribbon, and at parity of applied field conditions, the average speed is smaller since the ring curvature minimizes the rotational motion of the particles located close to the ring equator.

\section{CONCLUSION}

In summary, we have studied the assembly and controlled deformation of dipolar rings under external mag- 
netic fields. While in a previous article 35 the ring formation was reported, we demonstrate here real time manipulation of these fragile structures, and develop a theoretical model which explains the ring compression along the direction of the applied field. Our microrings are composed by synthesized hematite ellipsoids, similar self-assembled structures have been observed with other ferromagnetic particles on different length scales [19, 21, 41, 42]. Finally, the possibility to use these ellipsoids to entrap microscopic objects, like biological cells, shows the potentiality of this method for controlled encapsulation in fluidic environments.

\section{ACKNOWLEDGMENTS}

Acknowledgments F. M.P. and P. T. acknowledge support from the European Research Council (Project No. 335040). P. T. acknowledges support from Mineco (No. RYC-2011-07605 and No. FIS2013-41144-P) and AGAUR (Grant No. 2014SGR878). A. C. acknowledges support from National Research Programme No. 2014.10-4/VPP- 3/21. 


\section{Appendix A: Demonstration that Eq.1 holds for dipolar chain and rings}

Let us first consider the case of a chain of $N$ dipoles. Its dipolar energy reads

$$
U_{\text {chain }}=\sum_{i=1}^{N-1} \sum_{j=i+1}^{N}\left(\frac{\vec{m}_{i} \vec{m}_{j}}{r_{i j}^{3}}-\frac{3\left(\vec{r}_{i j} \cdot \vec{m}_{i}\right)\left(\vec{r}_{i j} \cdot \vec{m}_{j}\right)}{r_{i j}^{5}}\right),
$$

where $\vec{r}_{i j}=d(j-i) \vec{t} ; \vec{m}=m\left(e_{x}, 0, e_{z}\right) ; \vec{t}=(0,0,1)$, in the case when magnetic moment deviates from the axis of the chain. Then

$$
U_{\text {chain }}=\frac{m^{2}}{d^{3}} \sum_{i=1}^{N-1} \sum_{j=i+1}^{N} \frac{1}{(j-i)^{3}}\left(1-3(\vec{e} \cdot \vec{t})^{2}\right) .
$$

When $\vec{e} \cdot \vec{t}=1$, Eq. A2 transforms in the well-known relation for the energy of a straight chain

$$
U_{\text {chain }}^{0}=-\frac{2 m^{2}}{d^{3}} N K_{N},
$$

where

$$
K_{N}=\frac{1}{N} \sum_{l=1}^{N} \frac{N-l}{l^{3}} .
$$

To consider the deformation of the chain in an applied external field, the only important part in Eq.A2 is the one which depends on the orientation of the magnetic moment with respect to the axis of the chain:

$$
U_{\text {chain }}=\text { const }-\frac{3 m^{2}}{d^{3}} N K_{N}(\vec{e} \cdot \vec{t})^{2} .
$$

Since $M=m / d$ is the magnetization per unit length and $N d$ is the length of the chain, we have for the dipolar energy per unit length:

$$
U_{\text {chain }}=-\frac{3 M^{2} K_{N}}{d^{2}}(\vec{e} \cdot \vec{t})^{2}
$$

which is the second term of the right hand side of the Eq.1 of the paper. Note that in Eq.(A6) the constant term is omitted since the configuration of the ring is only determined by the orientation dependent term.

With more effort, we arrive at to a similar expression for the case of the ring. The positions of the particles in a ring with a radius $R$ are

$$
\vec{r}_{i}=R(\cos (2 \pi(i-1) / N), \sin (2 \pi(i-1) / N)),
$$

The magnetic moments form a constant angle with the azimuthal direction $\vec{e}_{\varphi}(i)=$ $(-\sin (2 \pi(i-1) / N), \cos (2 \pi(i-1) / N)), \quad$ where $\vec{e}_{i}=t_{r} \vec{e}_{r}(i)+t_{\varphi} \vec{e}_{\varphi}(i)$. For the dipolar energy per particle we have

$$
U_{1}=\sum_{i=2}^{N} m^{2}\left(\frac{\vec{e}_{i} \cdot \vec{e}_{1}}{r_{i 1}^{3}}-\frac{3\left(\vec{r}_{i 1} \cdot \vec{e}_{i}\right)\left(\vec{r}_{i 1} \cdot \vec{e}_{1}\right)}{r_{i 1}^{5}}\right)
$$

Since the magnetic moments are turned by the same angle $\vec{e}_{i} \cdot \vec{e}_{1}=\vec{e}_{\varphi}(i) \cdot \vec{e}_{\varphi}(1)=\cos (2 \pi(i-1) / N)$. Taking into account the relations

$$
\begin{array}{r}
\vec{r}_{i 1}=R(\cos (2 \pi(i-1) / N)-1, \sin (2 \pi(i-1) / N)) \\
r_{i 1}^{2}=4 R^{2} \sin ^{2}(\pi(i-1) / N) \\
\vec{r}_{i 1} \cdot \vec{e}_{1}=R\left(t_{\varphi} \sin (2 \pi(i-1) / N)+t_{r}(\cos (2 \pi(i-1) / N)-1)\right) \\
\vec{r}_{i 1} \cdot \vec{e}_{i}=R\left(t_{\varphi} \sin (2 \pi(i-1) / N)+t_{r}(1-\cos (2 \pi(i-1) / N))\right) \\
\left(\vec{r}_{i 1} \cdot \vec{e}_{1}\right)\left(\vec{r}_{i 1} \cdot \vec{e}_{i}\right)=R^{2}\left(\sin ^{2}(2 \pi(i-1) / N)-2 t_{r}^{2}(1-\cos (2 \pi(i-1) / N))\right),
\end{array}
$$

The dipolar energy per particle is given by

$$
U_{1}=-\frac{m^{2}}{8 R^{3}} \sum_{i=2}^{N} \frac{1+\cos ^{2}(\pi(i-1) / N)}{\sin ^{3}(\pi(i-1) / N)}+\frac{3 t_{r}^{2} m^{2}}{16 R^{3}} \sum_{i=2}^{N} \frac{1-\cos (2 \pi(i-1) / N)}{\sin ^{5}(\pi(i-1) / N)}
$$

Since $d=2 R \sin (\pi / N)$, the first part of $U_{1}, U_{10}$, coincides with a well-known expression used to describe the dipolar energy of a ring [27]:

$$
U_{\text {ring }}=\frac{N}{2} U_{10}=\frac{m^{2}}{d^{3}} \frac{N}{2} \sin ^{3}(\pi / N) \sum_{i=2}^{N} \frac{1+\cos ^{2}(\pi(i-1) / N)}{\sin ^{3}(\pi(i-1) / N)}
$$


The second part describes the increase of the dipolar energy due to the deviation of the direction of the magnetic moment from the azimuthal direction, which is supposed to be the same for all particles in the ring. This energy per unit length is

$$
e_{d}=-\frac{3 M^{2}}{4 d^{2}} \sin ^{3}(\pi / N) \sum_{i=2}^{N} \frac{1-\cos (2 \pi(i-1) / N)}{\sin ^{5}(\pi(i-1) / N)}(\vec{e} \cdot \vec{t})^{2}=-\frac{3 M^{2} K_{N}^{\prime}}{d^{2}}(\vec{e} \cdot \vec{t})^{2}
$$

where $\vec{e}$ are the unit vectors along the magnetic moments and $\vec{t}$ the tangent vectors to the ring all along its perimeter. The value of $K_{N}^{\prime}$ in this case is

$$
K_{N}^{\prime}=\frac{1}{4} \sin ^{3}(\pi / N) \sum_{i=2}^{N} \frac{1-\cos (2 \pi(i-1) / N)}{\sin ^{5}(\pi(i-1) / N)}
$$

and it is numerically very close to $K_{N}$.

\section{Appendix B: Derivation of Eqs.1 and 2 in the text}

The energy of the dipolar interactions in a ring made up of $N$ particles is

$$
E_{d}=-\frac{\pi}{2} M_{p}^{2} \frac{\pi}{6} d^{3} N K_{N}^{\prime}(\vec{e} \cdot \vec{t})^{2}
$$

Here, the angle between the vectors $\vec{e}$ and $\vec{t}$ is approximated to a constant value, assuming that it is the same all along the ring perimeter, and $M_{p}$ is the magnetization of the particles with diameters $d\left(m=M_{p} \pi d^{3} / 6\right)$. In order to calculate the energy of the ring, we introduce the magnetization per unit length, $M=m / d$. Then the dipolar energy per unit length $e_{d}=E_{d} / L(N=L / d)$ reads as

$$
e_{d}=-3 \frac{m^{2}}{d^{4}} K_{N}^{\prime}(\vec{e} \cdot \vec{t})^{2}
$$

or in an equivalent form

$$
e_{d}=-\frac{3 K_{N}^{\prime}}{d^{2}} M^{2}(\vec{e} \cdot \vec{t})^{2}
$$

Consequently, the demagnetization factor $\Delta N$ of a ring is $\Delta N=6 K_{N}^{\prime} / d^{2}$, and the total magnetic energy per unit length of the ring then reads as

$$
e_{m}=-M \vec{e} \cdot \vec{H}-\frac{1}{2} \Delta N M^{2}(\vec{e} \cdot \vec{t})^{2} .
$$

Assuming that the magnetic equilibrium is established faster than the mechanical equilibrium, the governing equations for the magnetic equilibrium can be written as

$$
\vec{K}_{\vec{e}} e_{m}=0
$$

being $\vec{K}_{\vec{a}}=\vec{a} \times \frac{\partial}{\partial \vec{a}}$. By considering an applied field with small amplitude, one can solve this equation using the power series, $\vec{e}=\vec{e}_{0}+\vec{e}_{1}+\vec{e}_{2}$. The zero order solution is $\vec{e}_{0}=\vec{t}$, and the condition $\vec{e}^{2}=1$ gives $\vec{e}_{0} \cdot \vec{e}_{1}=0$, and $\vec{e}_{0}$. $\vec{e}_{2}=-\vec{e}_{1}^{2} / 2$. Up to the second order term $\vec{e} \cdot \vec{t}=1+\vec{e}_{2} \cdot \vec{e}_{t}$, and the equation can be rewritten after a straightforward calculation (see similar derivation in [35]), as

$$
\vec{e}_{1}=-\frac{M H}{\Delta N M^{2}}[\vec{t} \times[\vec{t} \times \vec{h}]]
$$

The small amplitude hypothesis implies an applied field strength lower than the dipolar field created by the magnetic particle, given by $H_{d i p}=6 K_{N}^{\prime} m / d^{3}$. Using the values of $m, d$ given in the manuscript, the dipolar field obtained is $H_{d i p} \sim 0.6 \mathrm{Oe}$, which is actually larger than all the applied field strengths used in the experiments of ring deformation (Fig.2).

The torque per unit length can be written as, $\vec{K}=$ $-\vec{K}_{\vec{t}} e_{m}=\Delta N M^{2}(\vec{e} \cdot \vec{t})[\vec{t} \times \vec{e}]$. Here the second order approximation gives

$$
\vec{K}=\Delta N M^{2}\left[\vec{t} \times \vec{e}_{1}\right]+\Delta N M^{2}\left[\vec{t} \times \vec{e}_{2}\right]
$$

The equation of magnetic equilibrium up to the second order terms gives $-M\left[\vec{e}_{1} \times \vec{H}\right]-\Delta N M^{2}\left[\vec{e}_{2} \times \vec{t}\right]=0$, and by using Eq. B6] we obtain

$$
\vec{K}=M H[\vec{t} \times \vec{h}]-\frac{H^{2}}{\Delta N}(\vec{t} \cdot \vec{h})[\vec{t} \times \vec{h}]
$$

This corresponds to a linear energy density equal to

$$
e_{r}=-M H \vec{t} \cdot \vec{h}+\frac{H^{2}}{2 \Delta N}(\vec{t} \cdot \vec{h})^{2}
$$

\section{Appendix C: Derivation of Eq. 8 in the text}

Let us start from the energy functional (Eq.7):

$$
E=\frac{A}{2} \int\left(\frac{d \vartheta}{d l}\right)^{2} d l+\frac{H^{2}}{2 \Delta N} \int \cos ^{2} \vartheta d l
$$

where $\vec{t}=(\cos \vartheta, \sin \vartheta), d \vartheta / d l=-1 / R$ is the curvature of the ring, $l$ the natural parameter along the ring contour. 


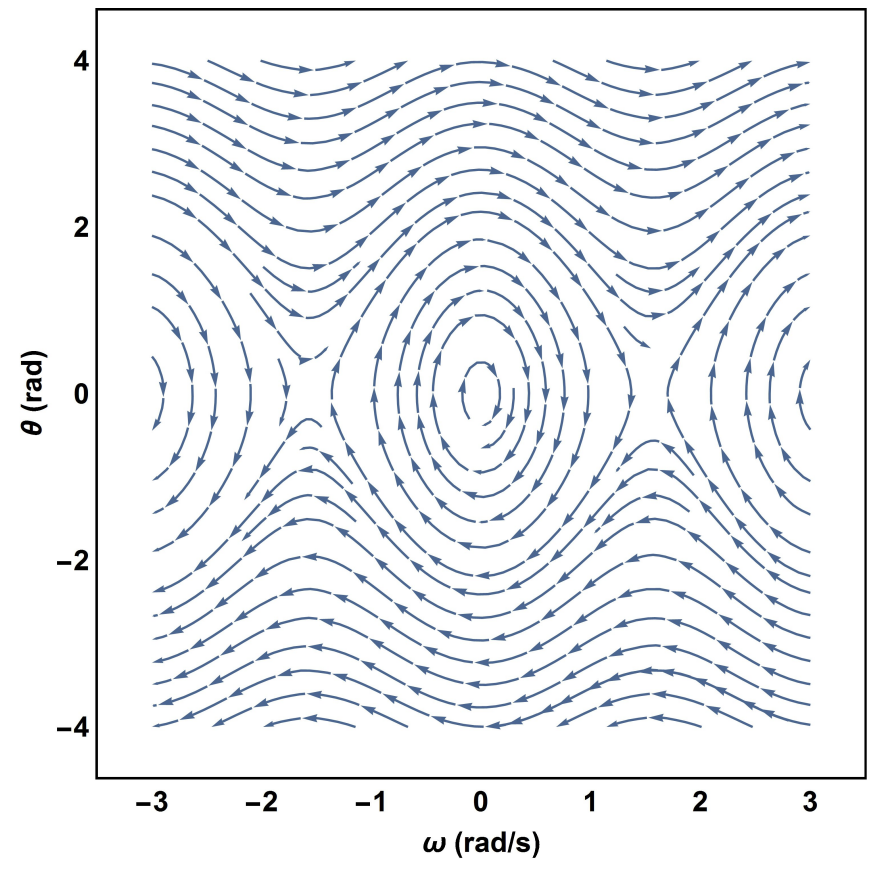

FIG. 5. Phase portrait of dynamical system given by Eq. C3 for $C_{m}=5$.

Minimizing the energy $\delta E=0$ gives the Euler-Lagrange equation (Eq.8):

$$
A \frac{d^{2} \vartheta}{d l^{2}}+\frac{H^{2}}{\Delta N} \cos \vartheta \sin \vartheta=0,
$$

where the angle $\theta$ changes from 0 to $2 \pi$ going around the ring with contour length $L$, due to the close form of the ring, which is supposed to be inextensible. Eq. C2 is equivalent to the dynamical system:

$$
\frac{d \vartheta}{d l}=\omega ; \frac{d \omega}{d l}=-\frac{H^{2}}{\Delta N} \cos (\vartheta) \sin (\vartheta),
$$

which has the following fixed points, $(\vartheta, \omega)=$ $(0,0) ;( \pm \pi, 0) ;( \pm \pi / 2,0) ;( \pm 3 \pi / 2,0) ; \ldots$ here, the first two are centers and second two are saddle points. Eqs $\mathrm{C3}$ may be put in dimensionless form by introducing the magnetoelastic number $C_{m}=\frac{H^{2} L^{2}}{\Delta N A}$. Fig.5 shows the phase portrait of the system described by Eqs C3 for $C_{m}=5$. There are two different phase trajectories. One corre- sponds to librations around the centers with limited variation of the angle $\vartheta$, while the second corresponds to the variation of the angle $\vartheta$ by $2 \pi$ along the contour. Only these trajectories correspond to possible solutions. The first integral of Eq. C2 is $C_{1}$ and is given by,

$$
\frac{A}{2}\left(\frac{d \vartheta}{d l}\right)^{2}+\frac{H^{2}}{2 \Delta N} \sin ^{2}(\vartheta)=C_{1} .
$$

Assuming a nonvanishing curvature of the ring, $C_{1}>$ $\frac{H^{2}}{2 \Delta N}$ and

$$
\frac{d \vartheta}{d l}=\sqrt{\frac{2}{A}} \sqrt{C_{1}-\frac{H^{2}}{2 \Delta N} \sin ^{2}(\vartheta)} .
$$

The solution of Eq. C5 reads as:

$$
\begin{gathered}
\int_{0}^{\vartheta} \frac{d \vartheta}{\sqrt{1-k^{2} \sin ^{2}(\vartheta)}}=\sqrt{\frac{2 C_{1}}{A}} l \\
\vartheta=a m\left(\sqrt{\frac{2 C_{1}}{A}} l, k\right)
\end{gathered}
$$

where $a m$ is the amplitude of elliptic integral and $k^{2}=$ $\frac{H^{2}}{2 \Delta N C_{1}}$. Since a change of the angle $\vartheta$ from 0 to $\pi / 2$ corresponds to a contour length $L / 4$, the parameter $k$ at given magnetoelastic number can be determined from the following equation:

$$
\sqrt{k^{2}} K(k)=\frac{1}{4} \sqrt{C_{m}} .
$$

where $K(k)$ is a complete elliptic integral of first kind and modulus $k$. The shape of the ring can be found by solving the set of equations:

$$
\frac{d x}{d l}=\cos (\vartheta) ; \frac{d y}{d l}=\cos (\vartheta) .
$$

Scaling the contour length $l$ by the length of the ring $L$ and using some properties of the elliptic functions, the previous equations may be written as:

$$
\frac{d x}{d l}=c n\left(\sqrt{\frac{C_{m}}{k^{2}}} l, k\right) ; \frac{d y}{d l}=s n\left(\sqrt{\frac{C_{m}}{k^{2}}} l, k\right),
$$

where $c n(u ; k)$ and $s n(u ; k)$ are the Jacobi elliptic functions of first and second kind.
[1] Y. Han and D. G. Grier, "Vortex rings in a constant electric field," Nature, 424, 267 (2003).

[2] C. Lutz, M. Kollmann, and C. Bechinger, "Single-file diffusion of colloids in one-dimensional channels," Phys. Rev. Lett., 93, 026001 (2004).

[3] H. Nagar and Y. Roichman, "Collective excitations of hydrodynamically coupled driven colloidal particles," Phys.
Rev. E, 90, 042302 (2014)

[4] P. J. Yunker, T. Still, M. A. Lohr, and A. G. Yodh, "Suppression of the coffee-ring effect by shape-dependent capillary interactions," Nature, 476, 308 (2011).

[5] W. Zhang, T. Yu, L. Liao, and Z. Cao, "Ring formation from a drying sessile colloidal droplet," AIP Advances, 3, 102109 (2013). 
[6] R. M. Erb, H. S. Son, B. Samanta, V. M. Rotello, and B. B. Yellen, "Magnetic assembly of colloidal superstructures with multipole symmetry," Nature, 457, 999 (2009).

[7] A. Ray, S. Aliaskarisohi, and T. M. Fischer, "Dynamics of self-assembly of flower-shaped magnetic colloidal clusters," Phys. Rev. E, 82, 031406 (2010).

[8] A. Snezhko and I. S. Aranson, "Magnetic manipulation of self-assembled colloidal asters," Nat. Materials, 10, 698703 (2011).

[9] Fernando Martinez-Pedrero and Pietro Tierno, "Magnetic propulsion of self-assembled colloidal carpets: Efficient cargo transport via a conveyor-belt effect," Phys. Rev. Applied, 3, 051003 (2015).

[10] A. Stradner, H. Sedgwick, F. Cardinaux, W. C. K. Poon, S. U. Egelhaaf, and P. Schurtenberger, "Equilibrium cluster formation in concentrated protein solutions and colloids," Nature, 432, 492 (2004).

[11] A. T. Skjeltorp, "One- and two-dimensional crystallization of magnetic holes," Phys. Rev. Lett., 51, 2306-2309 (1983)

[12] C. Goubault, P. Jop, M. Fermigier, J. Baudry, E. Bertrand, and J. Bibette, "Flexible magnetic filaments as micromechanical sensors," Phys. Rev. Lett., 91, 260802 (2003)

[13] W. Wen, F. Kun, K. F. Pál, D. W. Zheng, and K. N. Tu, "Aggregation kinetics and stability of structures formed by magnetic microspheres," Phys. Rev. E, 59, R4758 (1999).

[14] N. Vandewalle and S. Dorbolo, "Magnetic ghosts and monopoles," New J. Phys., 16, 013050 (2014).

[15] J. Yan, K. Chaudhary, S. C. Bae, J. A. Lewis, and S. Granick, "Colloidal ribbons and rings from janus magnetic rods," Nature Comm., 4, 1516 (2013).

[16] P. G. de Gennes and P. A. Pincus, "Pair correlations in a ferromagnetic colloid," Phys. Kondens. Mater., 11, 189 (1970).

[17] T. A. Prokopieva, V. A. Danilov, S. S. Kantorovich, and C. Holm, "Ground state structures in ferrofluid monolayers," Phys. Rev. E, 80, 031404 (2009).

[18] J. M. Tavares, J. J. Weis, and M. M. Telo da Gama, "Strongly dipolar fluids at low densities compared to living polymers," Phys. Rev. E, 59, 4388 (1999).

[19] F. Kun, W. Wen, K. F. Pál, and K. N. Tu, "Breakup of dipolar rings under a perpendicular magnetic field," Phys. Rev. E, 64, 061503 (2001).

[20] H. Morimoto, T. Maekawa, and Y. Matsumoto, "Statistical analysis of two-dimensional cluster structures composed of ferromagnetic particles based on a flexible chain model," Phys. Rev. E, 68, 61505 (2003).

[21] A. Ghazali and J.-C. Lévy, "Two-dimensional arrangements of magnetic nanoparticles," Phys. Rev. B, 67, 064409 (2003)

[22] P. D. Duncan and P. J. Camp, "Structure and dynamics in a monolayer of dipolar spheres," J. Chem. Phys., 121, 11322 (2004).

[23] A. Snezhko, I. S. Aranson, and W.-K. Kwok, "Structure formation in electromagnetically driven granular media," Phys. Rev. Lett., 94, 108002 (2005).

[24] T. Prokopyeva, V. Danilov, A. Dobroserdova, S. Kantorovich, and C. Holm, "Ground state structures in ferrofluid monolayers," J. Magn. Magn. Mat., 323, 1298 (2011).
[25] J. M. Tavares, L. Rovigatti, and F. Sciortino, "Quantitative description of the self-assembly of patchy particles into chains and rings," J. Chem. Phys., 137, 044901 (2012).

[26] L. Rovigatti, J. Russo, and F. Sciortino, "Structural properties of the dipolar hard-sphere fluid at low temperatures and densities," Soft Matter, 8, 6310 (2012).

[27] René Messina, Lara Abou Khalil, and Igor Stanković, "Self-assembly of magnetic balls: From chains to tubes," Phys. Rev. E, 89, 011202 (2014)

[28] René Messina, Khalil, and Igor Stanković, "Selfassembly of magnetic spheres in two dimensions: The relevance of onion-like structures," Europhys. Lett., 110, 46003 (2015).

[29] T. Sugimoto, M. M. Khan, and M. Muramatsu, "Preparation of monodisperse peanut-type a-fe2o3 particles from condensed ferric hydroxide gel," Colloids Surf. A, 70, 167 (1993).

[30] Pietro Tierno, Josep Claret, Francesc Sagués, and Andrejs Cēbers, "Overdamped dynamics of paramagnetic ellipsoids in a precessing magnetic field," Phys. Rev. E, 79, 021501 (2009).

[31] See the Supplemental Material at xxxx for supplementary videos and further theoretical derivation.

[32] A. Cebers, "Dynamics of a chain of magnetic particles connected with elastic linkers," J. of Phys.: Condensed Matter, 15, S1335 (2003).

[33] K. Erglis, R .Livanovics, and A. Cebers, "Three dimensional dynamics of ferromagnetic swimmer," J. Magn. Magn. Mat., 323, 1278 (2011).

[34] A. Cebers, "Flexible magnetic filaments," Curr. Opinion Coll. Interface Sci., 10, 167 (2005).

[35] F. Martinez-Pedrero, A. Cebers, and P. Tierno, "Orientational dynamics of colloidal ribbons self-assembled from microscopic magnetic ellipsoids," Soft Matter, 12, 3688 (2016).

[36] M. Belovs and A.Cebers, "Nonlinear dynamics of semiflexible magnetic filaments in an ac magnetic field," Phys. Rev. E, 73, 051503 (2006).

[37] K. Erglis, M. Belovs, and A.Cebers, "Flexible ferromagnetic filaments and the interface with biology," J. Magn. Magn. Mater., 321, 650 (2009).

[38] Pietro Tierno, Ramanathan Muruganathan, and Thomas M. Fischer, "Viscoelasticity of dynamically selfassembled paramagnetic colloidal clusters," Phys. Rev. Lett., 98, 028301 (2007).

[39] P. Tierno, R. Golestanian, I. Pagonabarraga, and F. Sagués, "Controlled swimming in confined fluids of magnetically actuated colloidal rotors," Phys. Rev. Lett., 101, 218304 (2008)

[40] N. Casic, N. Quintero, R. Alvarez-Nodarse, F. G. Mertens, L. Jibuti, W. Zimmermann, and T. M. Fischer, "Propulsion efficiency of a dynamic self-assembled helical ribbon," Phys. Rev. Lett., 110, 168302 (2013).

[41] H. Morimoto, K. Katano, and T. Maekawa, "Ring-chain structural transitions in a ferromagnetic particles system induced by a dc magnetic field," J. Chem. Phys., 131, 034905 (2009).

[42] A. Wei, T. Kasama, and R. E. Dunin-Borkowski, "Selfassembly and flux closure studies of magnetic nanoparticle rings," J. Mater. Chem., 21, 16686 (2011). 